\title{
Scattered Pilots and Virtual Carriers Based Frequency Offset Tracking for OFDM Systems: Algorithms, Identifiability, and Performance Analysis
}

\author{
Feifei Gao, Tao Cui, and Arumugam Nallanathan, Senior Member, IEEE
}

\begin{abstract}
In this paper, we propose a novel carrier frequency offset (CFO) tracking algorithm for orthogonal frequency division multiplexing (OFDM) systems by exploiting scattered pilot carriers and virtual carriers embedded in the existing OFDM standards. Assuming that the channel remains constant during two consecutive OFDM blocks and perfect timing, a CFO tracking algorithm is proposed using the limited number of pilot carriers in each OFDM block. Identifiability of this pilot based algorithm is fully discussed under the noise free environment, and a constellation rotation strategy is proposed to eliminate the $c$-ambiguity for arbitrary constellations. A weighted algorithm is then proposed by considering both scattered pilots and virtual carriers. We find that, the pilots increase the performance accuracy of the algorithm, while the virtual carriers reduce the chance of CFO outlier. Therefore, the proposed tracking algorithm is able to achieve full range CFO estimation, can be used before channel estimation, and could provide improved performance compared to existing algorithms. The asymptotic mean square error (MSE) of the proposed algorithm is derived and simulation results agree with the theoretical analysis.
\end{abstract}

Index Terms-Carrier frequency offset, OFDM, CFO ambiguity, constellation rotation, IEEE 802.11a.

\section{INTRODUCTION}

$\mathbf{O}$ RTHOGONAL frequency-division multiplexing [1], [2] is a promising candidate for next generation high-speed wireless communication systems due to its high data rate and robustness to frequency selective channels. It has been used in European digital audio/video broadcasting (DAB, DVB) [3], [4], high performance local area network (HIPERLAN) [5] and IEEE 802.11a wireless LAN standards [6]. In OFDM systems, it is well known that a CFO, caused by oscillators' mismatch or Doppler effects, destroys the subcarriers orthogonality, and results in a substantial bit error rate (BER) degradation [9]. Therefore, frequency synchronization should be performed before the channel estimation [7], [8] and the subsequent data detection.

Paper approved by Y. Li, the Editor for Wireless Communication Theory of the IEEE Communications Society. Manuscript received January 22, 2006; revised January 06, 2007 and February 13, 2007.

F. Gao is with the Department of Electrical \& Computer Engineering, National University of Singapore, 119260 (e-mail: feifeigao@nus.edu.sg).

A. Nallanathan is with the Division of Engineering, King's College London, United Kingdom (e-mail: arumugam.nallanathan@kcl.ac.uk).

T. Cui is with the Department of Electrical Engineering, California Institute of Technology, Pasadena, CA 91125, USA (e-mail: taocui@ caltech.edu).

Digital Object Identifier 10.1109/TCOMM.2008.060050.
Frequency synchronization usually contains two stages: the acquisition stage (coarse estimation) and the tracking stage (fine estimation). Several CFO acquisition methods have been proposed in [10]- [19]. In [10], the cyclic prefix (CP) in front of each OFDM block is exploited for an additive white Gaussian noise (AWGN) channel, and it is later extended for multi-path channel in [11]. Repeated training sequences are used in [12]- [14], and several improvements are made in [15][18]. Optimal training sequence design for CFO estimation over frequency selective channel has recently been proposed in [19], where the periodic structures are, again, exploited. Hence, almost all the methods in [10]- [19] use the periodic nature of the time domain signal, either by utilizing CP or by designing training sequences with repeated parts. However, using the periodic nature greatly reduces the CFO estimation range. Furthermore, all these methods, except [10], [11], are only applicable in CFO acquisition stage because consecutive training blocks are required, which are only available when transmission starts.

After the acquisition stage, there may exist a residue CFO, either because of the insufficient accuracy during the coarse estimation, or because of the time varying nature of the surrounding environment. The residue CFO, if not compensated, may still lead to performance degradation. Hence, many existing standards reserve a limited number of scattered pilot symbols ${ }^{1}$ in each OFDM blocks to improve the system robustness in different aspects. For example, in IEEE 802.11a WLAN standards [6], four pilots are placed at the subcarriers with indices $\{7,21,43,57\}$ for the purpose of combating the residue $\mathrm{CFO}$ and the phase noise.

In the tracking stage, the $\mathrm{CP}$ based method [11], the modification of [10], can still be used for residue CFO estimation. This method will be referred as Beek's method in this paper. However, the performance of Beek's method depends critically on the difference between the length of the $\mathrm{CP}$ and the channel length. Note that, in mobile environments, the channel length may not be a fixed value. Meanwhile, to achieve a high spectral efficiency, the $\mathrm{CP}$ length is not much greater than the channel length. Both these factors limit the performance of the $\mathrm{CP}$ based method. As a result, a better way

\footnotetext{
${ }^{1}$ The term "scattered pilot symbol" means that only limited number of pilots are inserted in each OFDM block. However, we do not assume a specific pattern on pilot positions.
} 
is to implement $\mathrm{CFO}$ tracking with the aid of scattered pilots available in existing OFDM standards. However, almost all the above mentioned CFO estimation methods [10]- [19] were developed based on preamble but not on data-pilot multiplexed symbols. To the best of the authors' knowledge, only the algorithms in [20]- [23] consider the scattered pilot tones. The method in [20] is referred as Classen\&Meyr's method in this paper. It is assumed that the channel impulse response (CIR) remains constant for two consecutive OFDM blocks over a slow fading channel. For a small CFO (much less than one subcarrier spacing) and a low signal-to-noise ratio (SNR), the inter carrier interference (ICI) induced by the CFO can be ignored as opposed to the large additive noise. Therefore, the CFO can be estimated by comparing the received symbols on the pilot carriers from the two consecutive OFDM blocks. However, this method fails to give good estimation when the residue CFO is large, and an error floor appears at high SNR. The methods in [21], [22] derive the best data-pilotmultiplexed scheme in terms of minimizing data-interference on pilot tones. Since the interference is minimized rather than being removed, these methods also meet error floors at high SNR. The method in [23] considers the statistic channel where the knowledge of the exact channel covariance matrix is required. However this requirement is not always fulfilled in the practical transmission if the environment is changing from time to time.

In this paper, we consider the deterministic channel and propose a novel CFO tracking algorithm that overcomes all the drawbacks in [20]- [22]. Assuming perfect timing, an algorithm, called $p$-algorithm, is developed using the scattered pilot carriers embedded in each OFDM block. Identifiability of $p$ algorithm is studied for the noise free case, and a constellation rotation strategy is proposed to eliminate one major type of the $\mathrm{CFO}$ ambiguity for widely used constellations. To further improve the performance of the CFO estimation and enhance the robustness to the $\mathrm{CFO}$ ambiguity, we consider using the virtual carriers existing in practical OFDM standards. For example, in IEEE 802.11a standards, the subcarriers with indices $\{0,27, \ldots, 37\}$ are set as virtual carriers, either to avoid the aliasing effect [24] or to be reserved for future use. The CFO estimation algorithm by exploiting virtual carriers, named $v$ algorithm in this paper, has been developed in [25]- [27]. A weighted algorithm, called $p v$-algorithm, is then proposed by exploiting both scattered pilots and virtual carriers. We show that in the $p v$-algorithm, the $p$-algorithm part increases the estimation accuracy, while the $v$-algorithm part reduces the outlier probability. Moreover, we derive the asymptotic mean square error (MSE) of our proposed algorithm, and the optimal weight in the $p v$-algorithm is given in a closedform. Besides tracking, the proposed algorithm can also be applied for $\mathrm{CFO}$ acquisition stage since it both provides full range $\mathrm{CFO}$ estimation and does not require the exact channel knowledge.

This paper is organized as follows. Section II presents the OFDM system model. Section III provides the proposed CFO tracking algorithm and discusses the ambiguity issues. Section IV gives the performance analysis of our proposed algorithm. Section V shows the simulation results, and conclusions are drawn in Section VI.

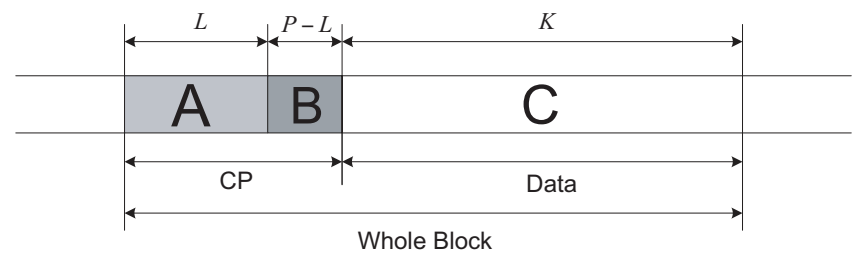

Fig. 1. Structure of an OFDM block.

Notations: The transpose, Hermitian, and inverse of a matrix $\mathbf{A}$ are denoted by $\mathbf{A}^{T}, \mathbf{A}^{H}$, and $\mathbf{A}^{-1}$, respectively; $\Re\{a\}$ and $\Im\{a\}$ denote the real and the imaginary parts of a complex scalar $a$, respectively; $|a|$ is the amplitude of $a$, and $|\mathcal{A}|$ is the cardinality of the set $\mathcal{A} ; \operatorname{tr}(\mathbf{A})$ is the trace of the matrix $\mathbf{A}$; $\mathrm{E}\{\cdot\}$ denotes the statistical expectation, and $\mathcal{I}$ is the integer set.

\section{Problem Formulation}

Let $K$ denote the number of subcarriers in one OFDM block. The index sets for pilot carriers and virtual carriers are denoted as $\mathcal{P}$ and $\mathcal{V}$, respectively. The transmitted symbol on the $k$ th subcarrier in the $m$ th OFDM block is

$s_{k}(m)=\left\{\begin{array}{ll}p_{k}(m) \in \mathcal{C}_{p} & k \in \mathcal{P} \\ 0 & k \in \mathcal{V} \\ d_{k}(m) \in \mathcal{C}_{d} & \text { otherwise }\end{array} \quad, \quad k=0, \ldots, K-1\right.$,

where $d_{k}(m)$ is the information symbol from the signal constellation $\mathcal{C}_{d}$, and $p_{k}(m)$ is the pilot symbol from the signal constellation $\mathcal{C}_{p}$. The power of the pilot symbols is normalized to unity, i.e., $\left|p_{k}(m)\right|=1$. Let

$$
\mathbf{s}(m)=\left[s_{0}(m), s_{1}(m), \ldots, s_{K-1}(m)\right]^{T}
$$

denote the $m$ th OFDM block in the frequency domain. After applying the inverse discrete Fourier transform (IDFT) to (2), the resulting time domain signal for the $m$ th OFDM block is given by

$$
\mathbf{z}(m)=\mathbf{F} \mathbf{s}(m)=\left[z_{0}(m), z_{1}(m), \ldots, z_{K-1}(m)\right]^{T},
$$

where $\mathbf{F}$ is the $K \times K$ normalized IDFT matrix with the $(a, b)$ th entry $F_{a, b}=\frac{1}{\sqrt{K}} e^{\frac{j 2 \pi(a-1)(b-1)}{K}}$. Each channel path is considered as a complex Gaussian random process, and the path delay is assumed to be integer multiple of the sampling interval $T_{s}$. Meanwhile, the maximum channel delay is upper bounded by $L T_{s}$, where $L$ is a positive integer. Note that no leakage is considered in this paper. The equivalent discrete channel vector can be represented as $\mathbf{h}=\left[h_{0}, \ldots, h_{L}\right]^{T}$, where $h_{l}$ denotes the $l$-th path of the channel, $l=0, \ldots, L$. For DFTbased OFDM, the CP $\left[z_{K-P}(m), \ldots, z_{K-1}(m)\right]$ is added in the front of $\mathbf{z}(m)$. The length of the CP, $P$, is chosen to be greater than or equal to $L$ to avoid the inter-block interference (IBI). The overall OFDM block of length $K_{s}=K+P$ is then transmitted with period $T_{s} K_{s}$. The structure of the whole OFDM block is shown in Fig. 1, where it is divided into three regions: $\mathbb{A}, \mathbb{B}, \mathbb{C}$. Symbols in region $\mathbb{A}$ are corrupted by IBI from the previous block. Region $\mathbb{B}$ represents the part in $\mathrm{CP}$ that is IBI free. Region $\mathbb{C}$ denotes information symbols $\mathbf{z}(m)$. All the OFDM blocks are placed sequentially in a data 
sequence $\left\{x_{t}\right\}, t=0,1, \ldots$ and is sent through a multi-path channel.

At the receiver, there usually exist both $\mathrm{CFO}$ and timing offset (TO), which must be estimated and compensated before the subsequent channel estimation and data detection. For simplicity we assume perfect timing synchronization. The received baseband signal can be expressed as

$$
r_{t}=e^{j 2 \pi t \phi} \sum_{l=0}^{L} h_{l} x_{t-l}+n_{t},
$$

where $n_{t}$ is the sample of zero-mean white complex Gaussian noise with variance $\sigma^{2}$, and $\phi$ is the CFO normalized by the whole bandwidth $1 / T_{s}$.

\section{FrequenCy OfFset TRACKING Method}

At the receiver, the $m$ th received block after the removal of the $\mathrm{CP}$ is given by

$$
\begin{aligned}
\mathbf{y}(m) & =\left[r_{(m-1) K_{s}+P}, \ldots, r_{m K_{s}-1}\right]^{T} \\
& =e^{j 2 \pi \phi\left((m-1) K_{s}+P\right)} \boldsymbol{\Omega}(\phi) \mathbf{F H s}(m)+\mathbf{n}(m),
\end{aligned}
$$

where $\boldsymbol{\Omega}(\phi)$ has the form of

$$
\boldsymbol{\Omega}(\phi)=\operatorname{diag}\left\{1, e^{j 2 \pi \phi}, \ldots, e^{j 2 \pi \phi(K-1)}\right\},
$$

and $\mathbf{H}$ is the diagonal matrix with its $(k, k)$ th element given by

$$
\mathbf{H}(k, k)=H_{k-1} \triangleq \sum_{l=0}^{L} h_{l} e^{-\frac{j 2 \pi(k-1) l}{K}} .
$$

\section{A. Pilot Based Tracking: p-Algorithm.}

Let $\tilde{\mathbf{s}}(m)=\left[\tilde{s}_{0}(m), \tilde{s}_{1}(m), \ldots, \tilde{s}_{K-1}(m)\right]^{T}$ denote the $K$ point normalized DFT of $\mathbf{y}(m)$. Then,

$$
\begin{aligned}
& \tilde{s}_{k}(m) e^{-j 2 \pi \phi\left((m-1) K_{s}+P\right)} \\
= & \frac{1}{K} \sum_{v=0}^{K-1} H_{v} s_{v}(m) e^{\frac{j(K-1) \pi(v+K \phi-k)}{K}} \\
& \times \frac{\sin (\pi(v+K \phi-k))}{\sin \left(\frac{\pi(v+K \phi-k)}{K}\right)}+\tilde{n}_{k}(m) \\
= & \frac{s_{k}(m) H_{k} e^{j(K-1) \pi \phi} \sin (\pi K \phi)}{K \sin (\pi \phi)}+\mathrm{ICI}(m)+\tilde{n}_{k}(m),
\end{aligned}
$$

where

$\mathrm{ICI}(m)=\sum_{v=0, v \neq k}^{K-1} \frac{\left.H_{v} s_{v}(m) e^{\frac{j(K-1) \pi(v+K \phi-k)}{K}} \sin \pi(v+K \phi-k)\right)}{K \sin \left(\frac{\pi(v+K \phi-k)}{K}\right)}$,

and $\tilde{n}_{k}(m)$ has the same distribution as $n_{t}$. For noise free case, $\tilde{s}_{k}(m)=H_{k} s_{k}(m)$ if $\phi=0$. A non-zero $\phi$ both introduces ICI and reduces the effective SNR to a factor of $\frac{e^{j(K-1) \pi \phi} \sin (\pi K \phi)}{K \sin (\pi K \phi)}$.

For a slow fading channel ${ }^{2}$, the CIR in $\nu=2$ consecutive blocks can be assumed static. Based on this fact, the Classen\&Meyr's method is developed by using a few number of pilots. In fact, Classen\&Meyr's method assumes a sufficiently small $\phi$ and a not high SNR, so that the ICI is much smaller than the noise and can thus be ignored. The $\mathrm{CFO}$ is then estimated as

$$
\begin{aligned}
& \hat{\phi}= \frac{1}{2 \pi K_{s}} \\
& \times \tan ^{-1} \frac{\sum_{k \in \mathcal{P}} \Im\left\{\tilde{s}_{k}^{*}(m) \tilde{s}_{k}(m+1) /\left(s_{k}^{*}(m) s_{k}(m+1)\right)\right\}}{\sum_{k \in \mathcal{P}} \Re\left\{\tilde{s}_{k}^{*}(m) \tilde{s}_{k}(m+1) /\left(s_{k}^{*}(m) s_{k}(m+1)\right)\right\}} .(10)
\end{aligned}
$$

Obviously, (10) is valid only when $\phi \ll \frac{1}{K}$. Therefore the accuracy of the coarse estimation during the $\mathrm{CFO}$ acquisition stage is crucial to the performance of (10) and even a small CFO variation in the tracking stage may cause the failure of the algorithm. There also exist other problems: 1) the estimation accuracy of (10) is limited by ignoring the ICI term. 2) At high SNR, since the ICI term is comparable to or even larger than the noise, the approximation in (10) is not valid any more.

In order to overcome all these drawbacks, we propose a new CFO tracking algorithm, still using several pilots but with an improved performance. Let $\varepsilon$ be the searching variable for the unknown CFO. After compensating $\mathbf{y}(m)$ by a diagonal matrix $\Omega(-\varepsilon)$, the symbol on the $k$ th subcarrier can be written as

$$
\begin{aligned}
& \tilde{s}_{k}(m) e^{-j 2 \pi \phi\left((m-1) K_{s}+P\right)} \\
& \frac{s_{k}(m) H_{k} e^{j(K-1) \pi(\phi-\varepsilon)} \sin (\pi K(\phi-\varepsilon))}{K \sin (\pi(\phi-\varepsilon))}+\mathrm{ICI}(m)+\bar{n}_{k}(m),
\end{aligned}
$$

where $\bar{n}_{k}(m)$ is the noise variable that distributes the same as $\tilde{n}_{k}(m)$. In the absence of noise, if $\varepsilon=\phi$, $\tilde{s}_{k}(m) e^{-j 2 \pi \varepsilon\left((m-1) K_{s}+P\right)}$ reduces to $s_{k}(m) H_{k}$, and $\tilde{s}_{k}(m+$ $1) e^{-j 2 \pi \varepsilon\left(m K_{s}+P\right)}$ is $s_{k}(m+1) H_{k}$. Since $H_{k}$ is same in two consecutive OFDM blocks, the metric

$$
\begin{aligned}
\sum_{k \in \mathcal{P}} \| \tilde{s}_{k}(m) e^{-j 2 \pi \varepsilon\left((m-1) K_{s}+P\right)} / s_{k}(m) \\
\quad-\tilde{s}_{k}(m+1) e^{-j 2 \pi \varepsilon\left(m K_{s}+P\right)} / s_{k}(m+1) \|^{2}
\end{aligned}
$$

is zero at $\varepsilon=\phi$. Note that, at least one of $H_{k}, k \in \mathcal{P}$ should not be zero, which is also assumed by Classen\&Meyr's method. Therefore, a CFO estimator ( $p$-algorithm) at the noisy

${ }^{2}$ Most frame based transmissions assume a slow fading channel, e.g. IEEE $802.11 \mathrm{a}[6]$

$$
\begin{aligned}
\hat{\phi} & =\arg \min _{\varepsilon} g_{p}(\varepsilon) \\
& =\arg \min _{\varepsilon} \sum_{k \in \mathcal{P}}\left\|e^{-j 2 \pi \varepsilon\left((m-1) K_{s}+P\right)} \mathbf{f}_{k}^{H} \boldsymbol{\Omega}(-\varepsilon) \mathbf{y}(m) / s_{k}(m)-e^{-j 2 \pi \varepsilon\left(m K_{s}+P\right)} \mathbf{f}_{k}^{H} \boldsymbol{\Omega}(-\varepsilon) \mathbf{y}(m+1) / s_{k}(m+1)\right\|^{2} \\
& =\arg \min _{\varepsilon}\left\|\mathbf{S}(m) \mathbf{F}_{p}^{H} \boldsymbol{\Omega}(-\varepsilon) \mathbf{y}(m)-\mathbf{S}(m+1) \mathbf{F}_{p}^{H} \boldsymbol{\Omega}(-\varepsilon) \mathbf{y}(m+1) e^{-j 2 \pi \varepsilon K_{s}}\right\|^{2} .
\end{aligned}
$$


case is proposed as (13) shown at the bottom of this page, where $\mathbf{f}_{k}$ is the $k$ th column of $\mathbf{F} ; \mathbf{F}_{p}$ is the $K \times|\mathcal{P}|$ matrix whose columns are obtained from $\mathbf{f}_{k}, k \in \mathcal{P}$; and $\mathbf{S}(m)$, $\mathbf{S}(m+1)$ are $|\mathcal{P}| \times|\mathcal{P}|$ diagonal matrices with the diagonal elements given by $1 / s_{k}(m), 1 / s_{k}(m+1), k \in \mathcal{P}$, respectively. If the channel is constant over $\nu>2$ blocks, the estimator (13) can be modified to

$$
\begin{aligned}
\hat{\phi}= & \arg \min _{\varepsilon} \sum_{u=0}^{\nu-2} \| \mathbf{S}(m+u) \mathbf{F}_{p}^{H} \boldsymbol{\Omega}(-\varepsilon) \mathbf{y}(m+u) \\
& -\mathbf{S}(m+u+1) \mathbf{F}_{p}^{H} \boldsymbol{\Omega}(-\varepsilon) \mathbf{y}(m+u+1) e^{-j 2 \pi \varepsilon K_{s}} \|^{2} .
\end{aligned}
$$

In the following discussion, we only focus on the case $\nu=2$ since $\nu$ is preferred to be smaller in order to track a varying CFO. An example for the case $\nu>2$ will be given in the simulation part.

In addition, it is interesting to find that (10) is equivalent to

$$
\begin{aligned}
\hat{\phi}=\arg \min _{\varepsilon} \| \mathbf{S}(m) \mathbf{F}_{p}^{H} \mathbf{y}(m) \\
\quad-\mathbf{S}(m+1) \mathbf{F}_{p}^{H} \mathbf{y}(m+1) e^{-j 2 \pi \varepsilon K_{s}} \|^{2}
\end{aligned}
$$

Obviously, (13) possesses a much more reasonable structure than (15), since it firstly rotates $\mathbf{y}(m), \mathbf{y}(m+1)$ by $\boldsymbol{\Omega}(-\varepsilon)$ and then estimate CFO by comparing the values on pilot carriers. On the contrary, the estimator (15) compares the values on pilot carriers from the direct DFT of $\mathbf{y}(m), \mathbf{y}(m+1)$, which may only give an acceptable approximation under certain conditions. A further benefit of (13) is that, it could possibly provide a full rage estimation of frequency offset. A first look on this point is from the fact that (13) contains the term $e^{-j 2 \pi \varepsilon}$, then $\phi \in(-0.5,0.5]$ is allowed. However, (15) only contains the term $e^{-j 2 \pi K_{s} \varepsilon}$ so the estimation range is limited to $\left(-0.5 / K_{s}, 0.5 / K_{s}\right]$.

\section{Remarks:}

- Estimator (13) is actually derived from observation, and it cannot be claimed as a maximum likelihood (ML) estimator. However, we may treat (13) as a suboptimal estimator since it gives an exact estimate under the noise free environment. Note that this property is not possessed by either (10) or (15).

- The exact knowledge of the channel model is critical for the channel statistics based algorithms [23], and the channel length plays a critical role for channel length based algorithm, e.g. Beek's method [10], [11]. Unfortunately, in practical communications, channel model may change for different environments, whose exact form is hard to obtain. Moreover, the exact channel length is difficult to estimate, either. In contrast, neither of these two factors is important for the proposed $p$-algorithm. The only assumption is that the CIR is constant for two consecutive OFDM blocks, which is relatively easier to be satisfied, especially in a slow fading channel.

\section{B. Identifiability For p-Algorithm}

Similar to [28], [29], we study the uniqueness of the estimator (13) under the noise free environment. The unknown CFO is assumed to be within the full region $(-0.5,0.5]$, and the trivial ambiguity $\hat{\phi}=\phi \pm b, b \in \mathcal{I}$ is excluded from the consideration (corresponding to a very large CFO). For the noise free case, (13) reduces to

$$
\hat{\phi}=\left\{\varepsilon \mid g_{p}(\varepsilon)=0\right\} .
$$

Obviously, the true $\mathrm{CFO} \phi$ is a solution to (16). The ambiguity appears if $\exists \bar{\phi} \neq \phi$ such that $g_{p}(\bar{\phi})=0$, which is equivalent to

$$
\begin{aligned}
0= & \sum_{v=0}^{K-1} H_{v} \underbrace{\frac{e^{\frac{j(K-1) \pi(v+K \Delta \phi-k)}{K}} \sin (\pi(v+K \Delta \phi-k))}{K \sin \left(\frac{\pi(v+K \Delta \phi-k)}{K}\right)}}_{\alpha_{v k}} \\
& \times \underbrace{\left(s_{v}(m) / s_{k}(m)-s_{v}(m+1) / s_{k}(m+1) e^{j 2 \pi \Delta \phi K_{s}}\right)}_{\beta_{v k}} \\
= & \sum_{v=0}^{K-1} H_{v} \alpha_{v k} \beta_{v k},
\end{aligned}
$$

for all $k \in \mathcal{P}$ and $\Delta \phi \neq 0$, where $\Delta \phi \triangleq \phi-\bar{\phi}$.

Case 1: $K \Delta \phi \notin \mathcal{I}$ : In this case, $\alpha_{v k} \neq 0$ for all $v$. The discussion is further divided into two subcases.

1) Not all $\beta_{v k}=0$ : The ambiguity happens when $\sum_{v} H_{v} \alpha_{v k} \beta_{v k}=0$. This type of ambiguity will be referred as $h$-ambiguity in this paper. Since $H_{v}$ is a linear combination of continuous complex random variables $h_{l}$, the probability for $h$-ambiguity is zero. Therefore, the $h$-ambiguity can be ignored.

2) All $\beta_{v k}=0$ : We call this kind of ambiguity as $d$ ambiguity. In order to avoid this type of ambiguity, the value on pilots can be properly designed such $\beta_{v k}$ is not zero for some $k \in \mathcal{P}$. For example, we can take $s_{k_{1}}(m)=1$, $s_{k_{1}}(m+1)=1$, while choose $s_{k_{2}}(m)=1, s_{k_{2}}(m+1)=-1$. Then, $\beta_{k_{1} k_{1}}, \beta_{k_{1} k_{2}}$ cannot be zero, simultaneously.

Case 2: $K \Delta \phi \in \mathcal{I}$ or more specifically, $K \Delta \phi \in \mathcal{I}_{K-1} \triangleq$ $\{1, \ldots, K-1\}^{3}$. Let $\tilde{v}_{k}=((k-K \Delta \phi) \bmod K)$. Obviously, $\tilde{v}_{k} \neq k$ when $\Delta \phi \neq 0$. In this case, $\alpha_{\tilde{v}_{k} k}=1$, and $\alpha_{v k}=0$, $\forall v \neq \tilde{v}_{k}$. The ambiguity happens if $\beta_{\tilde{v}_{k} k}=0$ or $H_{\tilde{v}_{k}}=0$. Since the latter can be equivalently considered as if the $\tilde{v}_{k}$ th carrier is a virtual carrier, we incorporate the discussion on $H_{\tilde{v}_{k}}=0$ into the discussion on $\beta_{\tilde{v}_{k} k}=0$, i.e.

$$
s_{\tilde{v}_{k}}(m) / s_{k}(m)-s_{\tilde{v}_{k}}(m+1) / s_{k}(m+1) e^{j 2 \pi \Delta \phi K_{s}}=0,(18)
$$$$
\text { for all } k \in \mathcal{P} \text {. }
$$

The discussion is divided into three subcases.

1) All $\tilde{v}_{k} \in \mathcal{P}$ : The ambiguity under this subcase is called $p$-ambiguity. Two different methods can be used to avoid the $p$-ambiguity. One is to choose the pilot index in a way that all pilot carriers are not equi-spaced. Then, the $p$-ambiguity cannot happen since any $K \Delta \phi \in \mathcal{I}_{K-1}$ could not make all $\tilde{v}_{k} \in \mathcal{P}$. However, since equi-spaced pilots can be used for optimal training in some OFDM systems [30] (if the number of the pilot carriers is greater than $L$ ), this approach to avoid $p$-ambiguity is not always recommended. The other way is to design the pilot symbols such that equation (18) does not hold for all $\tilde{v}_{k} \in \mathcal{P}$. If the pilot values are allowed to be arbitrary,

\footnotetext{
${ }^{3}$ We only need to consider this subset since $K \Delta \phi^{\prime}=K \Delta \phi+b K$ only provides a trivial ambiguity in $\Delta \phi^{\prime}=\Delta \phi+b$
} 
then the design is quite easier. However, we will restrict our special attention on pilot values belonging to $\{+1,-1\}$ as adopted in IEEE 802.11a standards [6]. The $p$-ambiguity can be avoided by properly consider at least three pilot carriers, say $\left\{k_{1}, k_{2}, k_{3}\right\}$. We can set $s_{k_{1}}(m)=s_{k_{1}}(m+1)=s_{k_{3}}(m)=$ $s_{k_{3}}(m+1)=1$, while taking $s_{k_{2}}(m)=1, s_{k_{2}}(m+1)=-1$. Then, (18) does not hold simultaneously for $k_{1}, k_{2}$ and $k_{3}$.

2) All $\tilde{v}_{k} \notin \mathcal{P}$ : This subcase can be further divided into two sub-subcases.

a) At least one $\tilde{v}_{k}, k \in \mathcal{P}$ does not belong to $\mathcal{V} \cup \mathcal{N}$, where $\mathcal{N}$ denotes the subcarrier index set for channel nulls. Without loss of generality, we denote this specific $k$ and $\tilde{v}_{k}$ as $k_{1}$ and $\tilde{v}_{k_{1}}$, respectively. The ambiguity under this sub-subcase is called $c$-ambiguity. Since the values of $s \tilde{v}_{k_{1}}(m), s_{\tilde{v}_{k_{1}}}(m+1)$ are selected from a finite alphabet, all the possible values of $\Delta \phi$ in (18) should belong to the set

$$
\begin{aligned}
& \Psi= \\
& \left\{\frac{1}{2 \pi K_{s}} \arg \left(\frac{s_{1}}{s_{2}}\right)+\frac{\omega}{K_{s}} \pm \frac{\iota}{K_{s}} \mid \forall s_{1}, s_{2} \in \mathcal{C}_{d}, \iota \in \mathcal{I}\right\},
\end{aligned}
$$

where

$$
\omega=\frac{1}{2 \pi} \arg \left(\frac{s_{k_{1}}(m+1)}{s_{k_{1}}(m)}\right)
$$

Let

$$
\mu \in \mathcal{A} \triangleq\left\{\frac{1}{2 \pi} \arg \left(\frac{s_{1}}{s_{2}}\right) \mid \forall s_{1}, s_{2} \in \mathcal{C}_{d}\right\}
$$

represent all the possible phase differences for a certain signal constellation $\mathcal{C}_{d}$. The $c$-ambiguity can be excluded if

$$
\frac{(\mu+\omega+\iota) K}{K_{s}} \notin \mathcal{I}_{K-1}, \quad \forall \mu \in \mathcal{A} .
$$

Note that, if pilots $s_{k_{1}}(m)$ and $s_{k_{1}}(m+1)$ could be chosen arbitrarily, (22) can be easily satisfied. However, this is not the case in practical transmissions, where the values of pilots are usually obtained from a constellation $\mathcal{C}_{p}$. We will, again, focus on the discussions when pilots can only be chosen from as $\{+1,-1\}$ as adopted in IEEE 802.11a standards. Similar discussions can be conducted for other pilot patterns by the interested readers themselves. Since the symmetric signal constellations are normally adopted, e.g., BPSK, QPSK, QAM, both $\frac{1}{2 \pi} \arg \left(\frac{s_{1}}{s_{2}}\right)$ and $\frac{1}{2 \pi}\left(\arg \left(\frac{s_{1}}{s_{2}}\right)+\pi\right)$ belongs to $\mathcal{A}$. Therefore, it is sufficient to consider only the case when $s_{k_{1}}(m)=s_{k_{1}}(m+1)=1$, which means $\omega$ is 0 . Then, the $c$-ambiguity can be excluded if

$$
\frac{(\mu+\iota) K}{K_{s}} \notin \mathcal{I}_{K-1}, \quad \forall \mu \in \mathcal{A} .
$$

Instead of designing the pilots values, we need to properly choose the signal constellation $\mathcal{C}_{d}$, and set the values of $K, K_{s}$. Note that, one value of $\mu$ must be zero, then $\frac{\iota K}{K_{s}} \notin \mathcal{I}_{K-1}$ is required. Therefore, $K$ and $K_{s}$ should at least be coprime numbers.

We give an example to illustrate the relationship between $c$-ambiguity and system parameters. For simplicity, we consider $K=16, P=5$ and the QPSK constellation.
Then, $\mathcal{A}$ is $\{0,0.25,0.5,0.75\}$, and the only solutions to $K \Delta \phi \in \mathcal{I}_{K-1}$ are

$$
\Delta \phi=\left\{\begin{array}{ll}
0.25 & \mu=0.25, \iota=5 \\
0.5 & \mu=0.5, \iota=10 \\
0.75 & \mu=0.75, \iota=15
\end{array} .\right.
$$

Meanwhile, if the symbol at the $\tilde{v}_{k}$ th carrier satisfies $s_{\tilde{v}_{k}}(m)=s_{\tilde{v}_{k}}(m+1) e^{j 2 \pi \Delta \phi K_{s}}$ for any $\Delta \phi$ in (24), then $\beta_{\tilde{v}_{k} k}=0$. In order to remove the ambiguity, we need to carefully reassign other values to $K, K_{s}$ such that (23) is satisfied. One thing to be mentioned is that, if the CFO can be restricted to the region $(-0.125,0.125]$, $c$-ambiguity is directly avoided, because the smallest $\Delta \phi$ in (24) is 0.25 . Note that, this ambiguity free region is five times wider than that of Classen\&Meyr's or Beek's method, which is only $(-0.5 / 21,0.5 / 21]$ (less than one subcarrier spacing).

b) All $\tilde{v}_{k}, k \in \mathcal{P}$ belong to $\mathcal{V} \cup \mathcal{N}$, where $\mathcal{N}$ denotes the subcarrier index set for channel nulls. We call this type of ambiguity as $n$-ambiguity. Since the index set for $\tilde{v}_{k}$ is actually a $K \Delta \phi$ cyclic shift from the set $\mathcal{P}$, we can properly design the number and the positions of pilot carriers such that at least one of $\tilde{v}_{k}$ does not belong to $\mathcal{V} \cup \mathcal{N}$ for any cyclic integer shift $K \Delta \phi \in \mathcal{I}_{k}$. A simple way is to choose $|\mathcal{P}|>|\mathcal{V}|+|\mathcal{N}|$. However, one may find better choices, if the CIR or at least the positions of channel nulls are known. It need to be mentioned that, the OFDM structure provided in IEEE 802.11a is almost $n$-ambiguity free, since the $\tilde{v}_{k}, k \in \mathcal{P}$ cannot simultaneously belong to $\mathcal{V}$, and $\mathcal{N}$ is an empty set with probability one.

3) Otherwise: The ambiguity for this subcase can be avoided by the methods in either of the previous two subcases.

Conclusion: Under the noise free condition, the CFO in the region $(-0.5,0.5]$ can be uniquely determined ${ }^{4}$ from the estimator (13) by properly designing system parameters, i.e., $\mathcal{P}, p_{k}(m), K, K_{s}$ and $\mathcal{C}_{d}$. However, it is hard to arrive at an explicit design for guaranteeing the absolute ambiguity avoidance for arbitrary systems because whether all types of ambiguities can be avoided, simultaneously, depends on quite a number of factors. Nevertheless, since we know the guidelines for each type of ambiguity free design, we could easily derive an ambiguity free solution for an explicit system.

Remark: The $p$-algorithm can be applied not only for CFO tracking but also for CFO acquisition because: 1) It could provide full range estimation; 2) It can be applied before channel estimation.

\section{Constellation Rotation: A Case Study for 802.11 a WLAN}

From the previous discussion, it is known that system parameters should be properly designed to eliminate the CFO estimation ambiguity. However, there exist several constraints that may bring inflexibility when designing some of these parameters. For example, $K$ is generally taken as $2^{p}$ so that the fast Fourier transform (FFT) operation can be implemented. Meanwhile, symmetric signal constellations are normally adopted, e.g., PSK, QAM.

\footnotetext{
${ }^{4} h$-ambiguity that happens with probability zero is ignored here.
} 
An example here follows the IEEE 802.11a standards, where the parameters are chosen as $K=64, P=16, K_{s}=$ $80, \mathcal{V}=\{0,27, \ldots, 37\}$, and $\mathcal{P}=\{7,21,43,57\}$. Every pilot takes the value of +1 or -1 . Obviously, the $d$-ambiguity can be readily removed by assigning \pm 1 to different pilots. Meanwhile $p$-ambiguity and $n$-ambiguity do not exist due to the position of pilot carriers ${ }^{5}$. We only need to deal with the $c$-ambiguity that happens when

$$
\frac{64}{80}(\mu+\iota)=\frac{4}{5}(\mu+\iota) \in\{1, \ldots, 63\} .
$$

It is readily seen that $\mu \in\{0,0.25,0.5,0.75\}$ or equivalently $\arg \left(\frac{s_{1}}{s_{2}}\right) \in\{0, \pi / 2, \pi, 3 \pi / 2\}$ are the only cases that may introduce $c$-ambiguity. Moreover, since $K, K_{s}$ are not coprime numbers, any signal constellation $\mathcal{C}_{d}$ could cause $c$-ambiguity. A way to resolve the $c$-ambiguity is to take double sets of modulations; namely, for OFDM block with odd index, we use signal constellation $\mathcal{C}_{d 1}$, whereas for OFDM blocks with even index, we use signal constellation $\mathcal{C}_{d 2}$. Let $\bar{s}_{1}$ be an arbitrary symbol in $\mathcal{C}_{d 1}$ and $\bar{s}_{2}$ be an arbitrary symbol in $\mathcal{C}_{d 2}$. Then, $\mathcal{C}_{d 1}, \mathcal{C}_{d 2}$ should be designed such that $\arg \left(\frac{\bar{s}_{1}}{\bar{s}_{2}}\right) \notin$ $\{0, \pi / 2, \pi, 3 \pi / 2\}$.

To keep the system BER performance unaffected, we suggest a constellation rotation scheme, i.e., $\mathcal{C}_{d 2}$ is a rotation from $\mathcal{C}_{d 1}$ by a proper angle $\theta$. For example, $\mathcal{C}_{d 1}$ is taken as QPSK, while $\mathcal{C}_{d 2}$ is taken as $\pi / 4$-QPSK. As shown in Fig. 2, $\arg \left(\frac{\bar{s}_{1}}{\bar{s}_{2}}\right)$ only belongs to $\{\pi / 4,3 \pi / 4,5 \pi / 4,7 \pi / 4\}$. Hence, the $c$-ambiguity can be totally removed for noise-free case. Actually, $\mathcal{C}_{d 2}$ can be rotated from $\mathcal{C}_{d 1}$ by an arbitrary $\theta \in(0, \pi / 2)$ for a QPSK constellation under noise free conditions. An optimal selection of $\theta$ for noisy environment is not strictly derived yet. However, due to the symmetry between the two constellations, a good choice may be $\theta=\pi / 4$ because the minimum $\arg \left(\frac{\bar{s}_{1}}{\bar{s}_{2}}\right)$ is maximized, which may result in a larger distance between $s_{\tilde{v}_{k}}(m)$ and $s_{\tilde{v}_{k}}(m+1) e^{j 2 \pi \Delta \phi K_{s}}$. We can also show that if $\theta=\pi / 4, c$-ambiguity in noise free case is also removed for higher order constellations, e.g. 16-QAM or 64-QAM.

Remark: The above discussion is established in the noise free environment. The ambiguity, or more properly called the outlier, may happen in the presence of noise. An example is given in the simulation part.

\section{Virtual Carriers Based Tracking: v-Algorithm}

The algorithms purely relying on virtual carriers have been studied in [25]- [27]. In this paper, we only quote their results and provide some necessary modifications. Under the perfect timing synchronization, the $\mathrm{CFO}$ estimator based on virtual carriers is written as

$$
\hat{\phi}=\arg \min _{\varepsilon} g_{v}(\varepsilon)=\arg \min _{\varepsilon} \sum_{q=m}^{m+1}\left\|\mathbf{F}_{v}^{H} \boldsymbol{\Omega}(-\varepsilon) \mathbf{y}(q)\right\|^{2},
$$

where $\mathbf{F}_{v}$ is the $K \times|\mathcal{V}|$ matrix whose columns are constructed from $\mathbf{f}_{k}, k \in \mathcal{V}$. In (26) we consider both the $m$ th and the $(m+$ 1)th received blocks to be consistent with the $p$-algorithm.

\footnotetext{
${ }^{5}$ Channel nulls on subcarriers are not considered here.
}

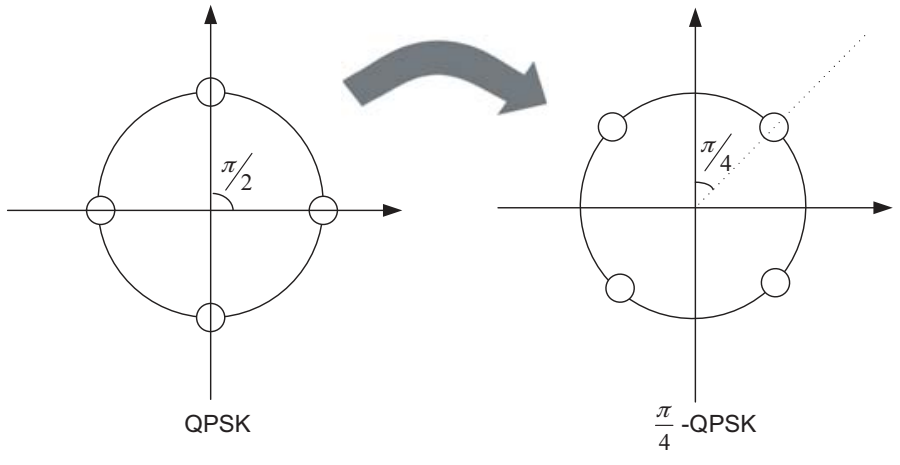

Fig. 2. Constellation Rotation for QPSK.

The identifiability study of CFO estimation in the presence of virtual carriers has been fully exploited in [31]. Interested readers are referred to [31] for detailed discussions.

\section{E. Co-Consideration: pv-Algorithm}

From previous subsections, we know that $p$-algorithm considers only pilot carriers, while $v$-algorithm considers only virtual carriers. Therefore the two algorithms work "independently" from each other. It is noted that, exploiting both pilot carriers and virtual carriers may offer several additional benefits:

1) Improve the estimation accuracy.

2) Reduce the probability of the ambiguity.

A reasonable combination of both $p$-algorithm and $v$-algorithm can be expressed as the weighted sum of the two corresponding cost functions. The combined cost function is given by

$$
g_{p v}(\varepsilon)=g_{p}(\varepsilon)+\gamma g_{v}(\varepsilon) .
$$

The first benefit by using (27) is because we exploit more information in $g_{p v}(\varepsilon)$ than purely relying on either $g_{p}(\varepsilon)$ or $g_{v}(\varepsilon)$. The second benefit can be explained in the absence of noise. Suppose $\exists \bar{\phi} \neq \phi$ such that $g_{p}(\bar{\phi})=0$. From intuition, since $g_{p}(\bar{\phi})$ and $g_{v}(\bar{\phi})$ are obtained through different approaches and possess different structures, the probability for $\bar{\phi}$ to be also the null point for $g_{v}(\varepsilon)$ is small. However, the true CFO $\phi$ must be the null point for both $g_{p}(\varepsilon)$ and $g_{v}(\varepsilon)$. Therefore, after the addition, the false null in either estimator will be compensated by the other estimator, leaving only the true $\phi$ being the null of $g_{p v}(\varepsilon)$. Note that, the ambiguity is still possible to happen once $\phi$ is the common null of both $g_{p}(\varepsilon)$ and $g_{v}(\varepsilon)$. However, the probability may be greatly reduced compared with using either estimator. Furthermore, to eliminate the ambiguity for $p v$-algorithm, we only need to eliminate the ambiguity for $p$-algorithm (as in subsection III-B), or to eliminate the ambiguity for $v$-algorithm [31].

An example regarding the second benefit is shown here. For simplicity, the parameters are taken as $L=3, K=16$, $P=5, \phi=0.25, \gamma=1, \mathcal{P}=\{11\}, \mathcal{V}=\{13,14,15\}$. Symbols are generated from QPSK constellations and pilots are selected as $p_{11}(m)=p_{11}(m+1)=+1$. We also set $H_{12}=0$. From [31], the ambiguity for $v$-algorithm takes place at the point $\varepsilon=0.1875$. For $p$-algorithm, the $c$-ambiguity may happen at the point $\varepsilon \in\{0,-0.25,0.5\}$ as shown in (24). The $p$-ambiguity does not exist since there is only one 


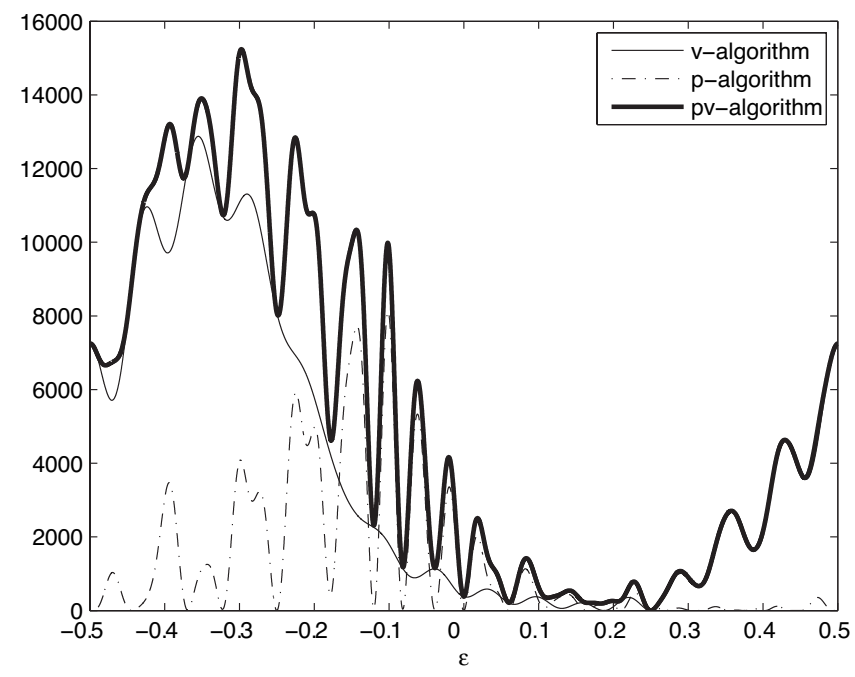

Fig. 3. CFO pattern for $p$-algorithm, $v$-algorithm and $p v$-algorithm.

pilot carrier. The $d$-ambiguity only happens when the two OFDM blocks are exactly the same and can be ignored in this example. Meanwhile, the $n$-ambiguity takes on place at $\varepsilon \in\{0.3750,0.4375,0.5\}$ because of the virtual carriers, and at $\varepsilon=0.3125$ because of the channel null. It is seen that the $n$-ambiguity, caused by the channel null, in $p$ algorithm shifts to the opposite direction as that in $v$-algorithm, i.e., $0.1875=0.25-1 / 16$ while $0.3125=0.25+1 / 16$. Hence, there is no common null for both $p$-algorithm and $v$-algorithm.

The cost function (13), (26), (27) of $p$-algorithm, $v$ algorithm, and $p v$-algorithm in the absence of noise are shown in both Fig. 3 and Fig. 4. From these two figures, although both $p$-algorithm and $v$-algorithm suffer from their respective ambiguity, the $p v$-algorithm has a unique null at the true CFO value, because the false null of either algorithm is compensated by the other algorithm.

\section{F. Ways to Obtain $\hat{\phi}$ from $g_{p}(\varepsilon)$ and $g_{p v}(\varepsilon)$}

The direct way to estimate $\phi$ from either $g_{p}(\varepsilon)$ or $g_{p v}(\varepsilon)$ is the one dimensional searching. However, the complexity of such kind of searching, although acceptable in some applications, e.g., direction of arrival (DOA) estimation [32], is too high to be implemented in other real time applications. To avoid computationally expensive searching, several alternatives are considered. We will illustrate these methods for $p v$-algorithm whereas the extensions to $p$-algorithm would be an easier job.

1) FFT based method: The estimator (27) can be expanded as

$$
g_{p v}(\varepsilon)=\rho_{0}+2 \Re\left\{\sum_{i=1}^{K+K_{s}} \rho_{i} e^{-j 2 \pi i \varepsilon}\right\},
$$

where $\rho_{i}$ can be obtained from (27) straightforwardly. From [14], we know that the minimization of the cost function can be achieved through the fast Fourier transform (FFT).

2) Polynomial Rooting: Polynomial rooting for a single $\varepsilon$ has been proposed in [27]. This method is able to guarantee the global minimum of $g_{p v}(\varepsilon)$, and its complexity can be approximated by $O\left(\left(K+K_{s}\right)^{3}\right)$. Compared with the FFT

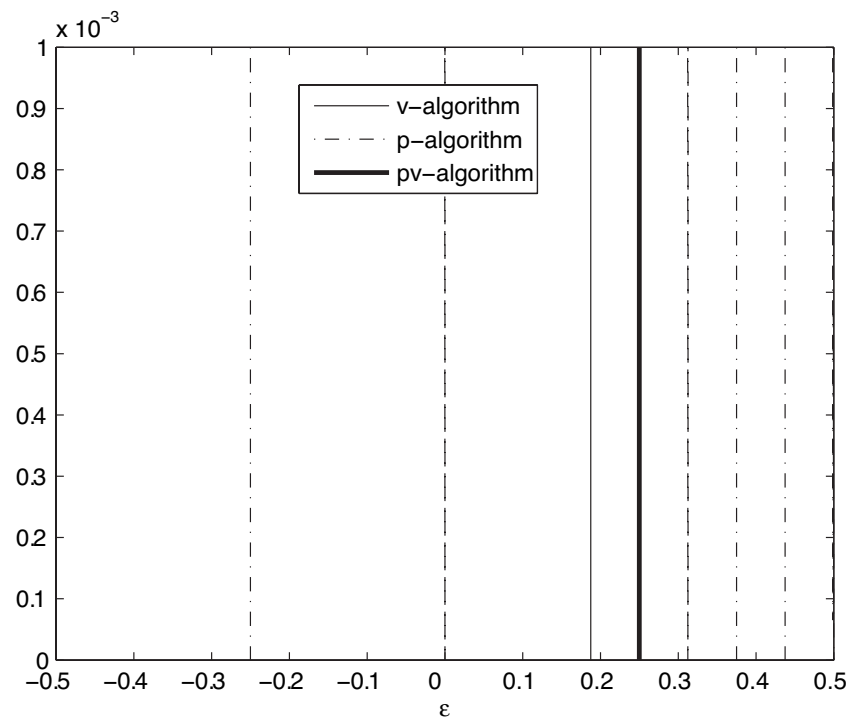

Fig. 4. Scope-enlarged CFO pattern.

based searching, the polynomial rooting is recommended for smaller $K$ and $K_{s}$.

3) Adaptive method: Adaptive algorithm can be utilized in certain cases in order to reduce the computational complexity. However, the major drawback of adaptive method is that, the initial point is critical to guarantee a global minimum.

\section{Performance Analysis}

For the ease of analysis, we assume that all pilots are taken as +1 . Assuming SNR $\gg 1$, the expectation and the variance of the proposed estimator can be approximated by [14]

$$
\begin{aligned}
& \mathrm{E}_{p v}\{\hat{\phi}\} \cong \phi-\frac{\mathrm{E}\left\{\dot{g}_{p v}(\phi)\right\}}{\mathrm{E}\left\{\ddot{g}_{p v}(\phi)\right\}}=\phi-\frac{\mathrm{E}\left\{\dot{g}_{p}(\phi)+\gamma \dot{g}_{v}(\phi)\right\}}{\mathrm{E}\left\{\ddot{g}_{p}(\phi)+\gamma \ddot{g}_{v}(\phi)\right\}}, \\
& \operatorname{Var}_{p v}\{\hat{\phi}\} \cong \frac{\mathrm{E}\left\{\left[\dot{g}_{p v}(\phi)\right]^{2}\right\}}{\left[\mathrm{E}\left\{\ddot{g}_{p v}(\phi)\right\}\right]^{2}}=\frac{\mathrm{E}\left\{\left[\dot{g}_{p}(\phi)+\gamma \dot{g}_{v}(\phi)\right]^{2}\right\}}{\left[\mathrm{E}\left\{\ddot{g}_{p}(\phi)+\gamma \ddot{g}_{v}(\phi)\right\}\right]^{2}}
\end{aligned}
$$

where $\dot{g}(\phi)$ and $\ddot{g}(\phi)$ represent the first and the second order derivatives of $g(\varepsilon)$ at $\phi=\varepsilon$. As derived in the Appendix, (29) and (30) can be simplified as

$$
\begin{aligned}
& \mathrm{E}_{p v}\{\hat{\phi}\} \cong \phi, \\
& \operatorname{Var}_{p v}\{\hat{\phi}\} \cong \frac{\sigma^{2}}{8 \pi^{2}} \frac{2 Z_{p}+\gamma^{2} Z_{v}}{\left(Z_{p}+\gamma Z_{v}\right)^{2}},
\end{aligned}
$$

where

$$
\begin{aligned}
& Z_{p}=\left\|\mathbf{P}_{p}\left(\mathbf{D} \Delta \boldsymbol{\eta}-K_{s} \boldsymbol{\eta}(m+1)\right)\right\|^{2}, \\
& Z_{v}=\sum_{q=m}^{m+1} \boldsymbol{\eta}^{H}(q) \mathbf{D} \mathbf{P}_{v} \mathbf{D} \boldsymbol{\eta}(q),
\end{aligned}
$$

and all the variables are defined in the Appendix. Note that $\mathbf{P}_{p} \boldsymbol{\eta}(m+1)$ is the term only related to the variable $H_{k}, k \in \mathcal{P}$, and $\operatorname{Var}_{p v}\{\hat{\phi}\}$ is not related to the specific CFO value. Meanwhile, the CFO estimation variances by using either $g_{p}(\varepsilon)$ or $g_{v}(\varepsilon)$ are

$$
\begin{aligned}
\operatorname{Var}_{p}\{\hat{\phi}\} \cong \frac{\sigma^{2}}{4 \pi^{2} Z_{p}}, \\
\operatorname{Var}_{v}\{\hat{\phi}\} \cong \frac{\sigma^{2}}{8 \pi^{2} Z_{v}},
\end{aligned}
$$


respectively. It is interesting to find that

$$
\begin{aligned}
\left.\operatorname{Var}_{p v}\{\phi\}\right|_{\gamma=0} & =\operatorname{Var}_{p}\{\phi\}, \\
\left.\operatorname{Var}_{p v}\{\phi\}\right|_{\gamma \rightarrow \infty} & =\operatorname{Var}_{v}\{\phi\} .
\end{aligned}
$$

Therefore the value of $\gamma$ controls the effective part of each single estimator.

A nice property of weighted sum of $p$-algorithm and $v$ algorithm is that, the closed form of the optimal weight $\gamma$ can be obtained regardless of all other parameters. Taking the derivative of (32) with respect to $\gamma$, we arrive at that, the minimum value of $\operatorname{Var}_{p v}\{\hat{\phi}\}$ is always achieved at $\gamma=2$.

\section{Numerical RESUlts FOR CFO Estimation}

In this section, we examine the performance of the proposed estimators under various scenarios. All parameters are taken from IEEE 802.11a standards. The 4-ray channel model with an exponential power delay profile [33]

$$
E\left\{\left|h_{l}\right|^{2}\right\}=\kappa \exp (-l / 10), \quad l=0, \ldots, L
$$

is used where $\kappa$ is the coefficient to normalize the overall channel gain. Each channel path is complex Gaussian. The normalized estimation mean square errors (NMSE) is defined as

$$
\mathrm{NMSE}=\frac{1}{N} \sum_{i=1}^{N} \frac{\left(\hat{\phi}_{i}-\phi\right)^{2}}{\phi^{2}},
$$

where $N=100$ Monte-Carlo runs are taken for average.

\section{A. CFO Less than Subcarrier Spacing}

In this example, different CFOs are taken from the region $(-0.5 / 64,0.5 / 64]$. The performance of $p$-algorithm, $v$ algorithm, $p v$-algorithm, Classen\&Meyr's method and Beek's method are compared. We assume that the estimated channel length is $\hat{L}=12$ in order to give a fair comparison between the Beek's method and our proposed algorithms ${ }^{6}$. Furthermore, QPSK constellation is used for all OFDM blocks. The NMSEs versus SNR for different algorithms are shown in Fig. 5, and the theoretical results for $p$-algorithm, $v$-algorithm, and $p v$ algorithms are given as well. As can be seen from the figure, Classen\&Meyr's method can give a relatively satisfying performance at lower SNR with a normalized CFO 0.1 subcarrier spacing. However, at high SNR, the Classen\&Meyr's method has an error floor. Meanwhile, even when CFO is as small as 0.25 subcarrier spacing, the Classen\&Meyr's method fails, because the ICI term cannot be ignored any more. On the contrary, since our $p$-algorithm does not make any approximation, it does not have an error floor and is also valid for a large CFO value. It is also noted that, Beek's method, $p$-algorithm and $p v$-algorithm give comparable performance. However, our major concern is that the performance of Beek's method is greatly affected by the channel length, or the estimate of the channel length. For example, if $L=16$, or if $L<16$ but the estimate $\hat{L}=16$ due to the power leakage, then the Beek's method can not even be applied. For $v$-algorithm, although no error floor is met, the performance is much worse than either

\footnotetext{
${ }^{6}$ The length of the region $\mathbb{B}$ should be the same as $|\mathcal{P}|$ for fairness [11].
}

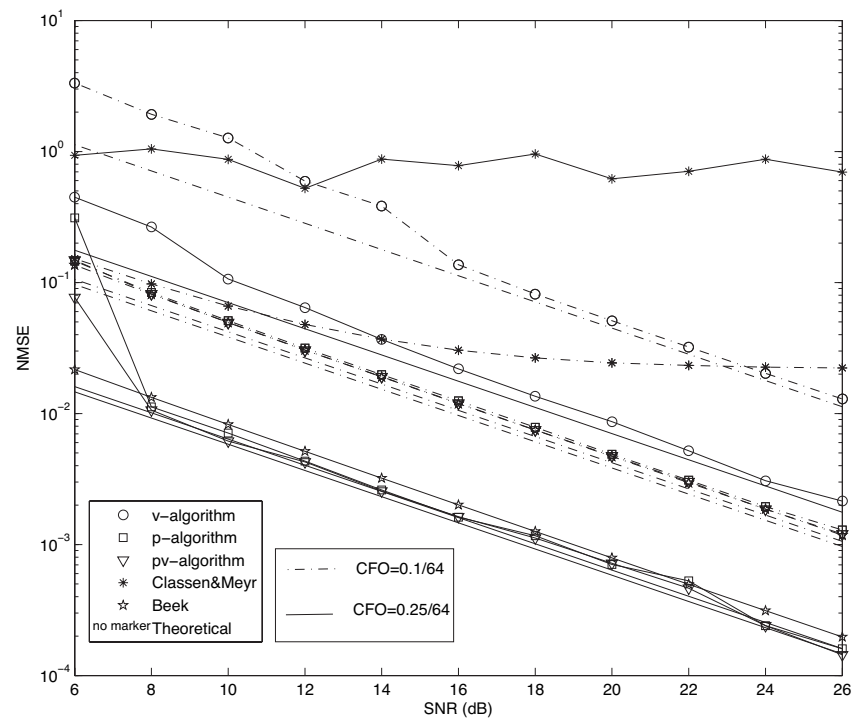

Fig. 5. NMSEs versus SNR for different CFO estimation algorithm: CFO smaller than subcarrier spacing.

the $p$-algorithm or the $p v$-algorithm. This is because that $v$ algorithm only consider the orthogonality between subcarriers and is actually a blind type CFO estimation method. From intuition, pilot aided algorithm outperforms $v$-algorithm. Note that there are three dashed lines without any marker. These lines, ordered from top down, represent the theoretical NMSE of $v$-algorithm, $p$ algorithm, and $p v$-algorithm for CFO equaling to 0.1 subcarrier spacing, respectively. Similar discussions hold for three solid lines in the figure for $\mathrm{CFO}$ equaling 0.25 subcarrier spacing. We find that the numerical performance of $p$-algorithm and $p v$-algorithm agree with theoretical analyzes very well, which verifies our analytical studies.

We also verify the optimality of the choice for $\gamma$ in Fig. 6, which shows the NMSE of $p v$-algorithm versus different value of $\gamma$ at $\mathrm{SNR}=15 \mathrm{~dB}$. Clearly, $\gamma=2$ is the optimal weight, which agrees with the theoretical result. Since the $p$ algorithm is the dominant component of the $p v$-algorithm, the performance of the $p v$-algorithm does not depend critically on the choice of $\gamma$. From Fig. 6, we find that the NMSE value does not change too much within the region $\gamma \in[0,5]$.

\section{B. CFO Larger than Subcarrier Spacing}

One important contribution of our proposed algorithm is its applicability for CFO greater than subcarrier spacing. In this example, we consider the performance of $p$-algorithm, $v$ algorithm, $p v$ algorithm. Note that, Classen\&Meyr's method and Beek's method are not included here because they are not applicable for this scenario. The constellation schemes with and without rotation are compared. For the former scheme, $\mathcal{C}_{d 1}$ is QPSK and $\mathcal{C}_{d 2}$ is $\pi / 4$-QPSK, while for the latter scheme, QPSK constellation is used for all OFDM blocks. The CFO is taken as large as 0.25 of total bandwidth, which is 16 subcarrier spacings. NMSEs versus SNR are shown in Fig. 7. It is seen from Fig. 7 that, the $p v$-algorithm is about $12 \mathrm{~dB}$ better than $v$-algorithm and gives accurate estimation over all SNRs. However, it is also noted that the $p$-algorithm with constellation rotation cannot yield good performance 


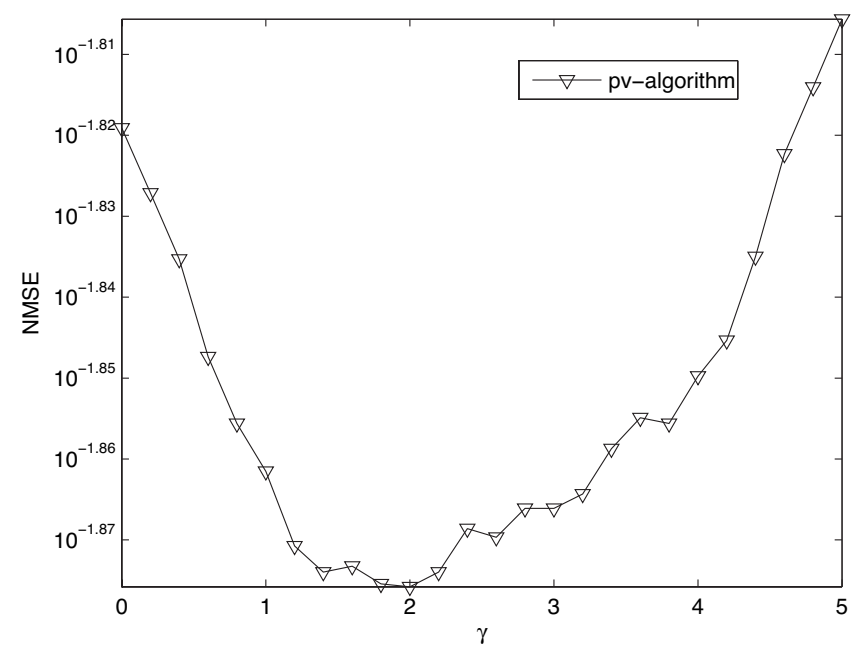

Fig. 6. NMSEs for $p v$-algorithm under different weight $\gamma$.

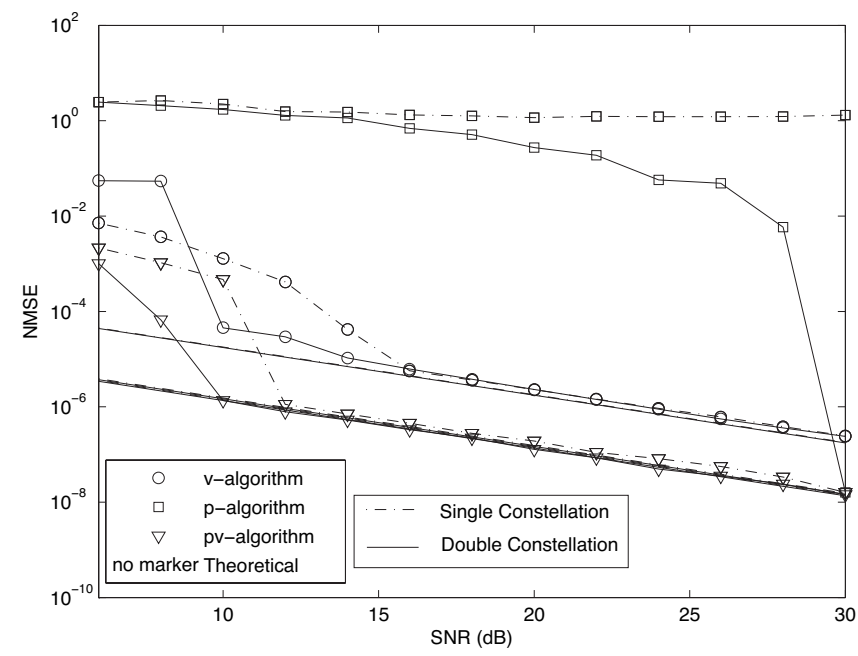

Fig. 7. NMSEs versus SNR for different $\mathrm{CFO}$ estimation algorithm: $\mathrm{CFO}$ larger than subcarrier spacing.

for $\mathrm{SNR}<30 \mathrm{~dB}$, and the $p$-algorithm without constellation rotation fails at all SNR. In the simulations, we have observed that several Monte-Carlo runs give outlier on CFO estimation. From Fig. 3, we see that the noiseless cost function $g_{p}(\varepsilon)$ is close to zero at several locations. If noise is present, those close-to-zero points may yield the minimum value in the cost function $g_{p}(\varepsilon)$, which causes CFO outlier. In section III-B, we only provide the discussion on ambiguity elimination for noise free environment. If the noise is present, the outlier may happen. Nevertheless, our $p v$-algorithm benefits from both algorithms. The $p$-algorithm part increases the estimation accuracy while the $v$-algorithm part reduces the outlier probability.

A reasonable way to evaluate the advantages of the constellation rotation scheme is to consider the CFO outlier probability (CFOOP), which is defined as

$$
\mathrm{CFOOP}=\frac{\text { the number of runs with outlier }}{\text { the total number of Monte Carlo runs }} .
$$

where the outlier in the presence of the noise may be defined as:

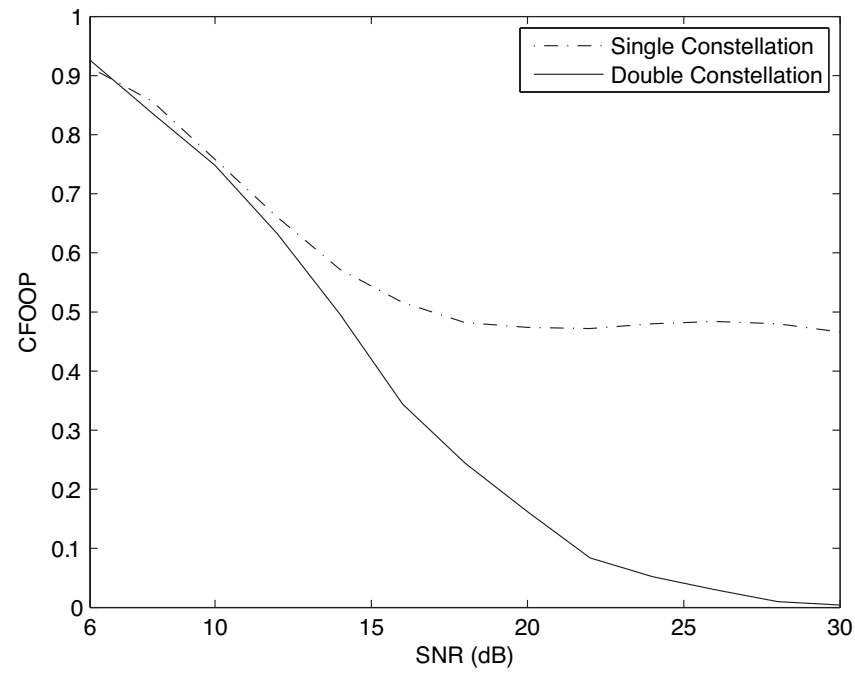

Fig. 8. CFOOP versus SNR for $p$-algorithm: Comparison of two modulation schemes.

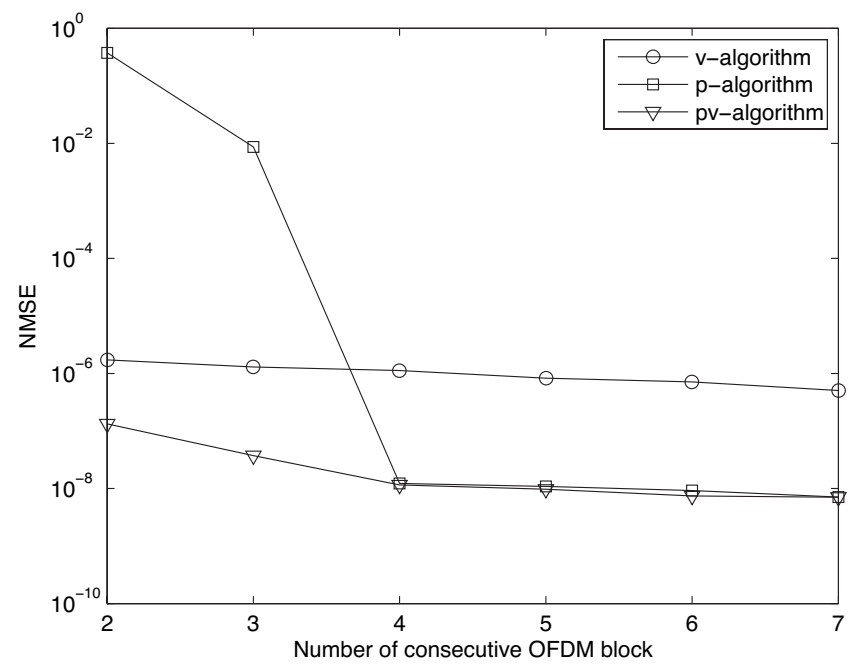

Fig. 9. NMSEs versus number of the consecutive OFDM blocks: CFO lager than subcarrier spacing.

Definition: In noisy environment, the outlier occurs if the estimated $\hat{\phi}$ stays outside the region $[\phi-0.5 / K, \phi+0.5 / K]$.

The comparison of CFOOP for $p$-algorithm with different constellation schemes is shown in Fig. 8. Clearly, the CFOOP is reduced to zero at high SNR using the constellation rotation scheme whereas it meets a floor at high SNR without constellation rotation. As analyzed in subsection III-B, the $c$-ambiguity for non-rotation scheme can never be removed, which consequently introduces non-zero CFOOP all the time.

It has been shown that the proposed $p$-algorithm, $p v$ algorithm can be readily modified when CIR and CFO is constant over more than two consecutive OFDM blocks. Using the same parameters as in Fig. 7, we show the NMSEs of different algorithms versus the number of the consecutive OFDM blocks used for CFO estimation in Fig. 9. The SNR is fixed at $20 \mathrm{~dB}$. From Fig. 9 we find that the outlier of $p$-algorithm in noisy environment can also be "removed" by increasing the number of blocks used. Actually, the probability of the occurrence of the outlier is reduced when more OFDM blocks are used. 


\section{CONCLUSIONS}

In this paper, a novel CFO tracking method was developed for practical OFDM systems. The proposed algorithm considers both pilot carriers and virtual carriers, hence is compatible to most practical standards. The ambiguity for pilot based algorithm was studied and several approaches to remove different types of the ambiguity were discussed. Since many parameters are already fixed in existing standards, a constellation rotation scheme was suggested to remove the $c$ ambiguity effect. Performance of our proposed algorithms was analyzed, and numerous simulation results were conducted to validate the theoretical results. From the simulation results, we found that the pilot based algorithm increases the estimation accuracy while the virtual carrier based algorithm reduces the outlier probability. Therefore, the proposed algorithm is not only an effective method for CFO tracking but also is applicable for $\mathrm{CFO}$ acquisition.

\section{ACKNOWLEDGEMENT}

The authors would like to thank the anonymous reviewers for their critical comments that greatly improved this paper.

\section{APPENDIX}

DERIVATION OF (31) AND (32)

Define new vectors

$$
\begin{aligned}
\breve{\mathbf{y}}(m) & =e^{-j 2 \pi \phi\left((m-1) K_{s}+P\right)} \boldsymbol{\Omega}(-\phi) \mathbf{y}(m) \\
& =\underbrace{\mathbf{F H} \mathbf{s}(m)}_{\boldsymbol{\eta}(m)}+\underbrace{e^{-j 2 \pi \phi(m-1) K_{s}} \boldsymbol{\Omega}(-\phi) \mathbf{n}(m)}_{\breve{\mathbf{n}}(m)}, \\
\breve{\mathbf{y}}(m+1) & =e^{-j 2 \pi \phi\left(m K_{s}+P\right)} \boldsymbol{\boldsymbol { \Omega } ( - \phi ) \mathbf { y } ( m + 1 )} \\
& =\underbrace{\mathbf{F H} \mathbf{s}(m+1)}_{\boldsymbol{\eta}(m+1)}+\underbrace{e^{-j 2 \pi \phi m K_{s}} \boldsymbol{\Omega}(-\phi) \mathbf{n}(m)}_{\breve{\mathbf{n}}(m+1)} .
\end{aligned}
$$

Obviously, $\breve{\mathbf{n}}(m)$ has the same distribution as that of $\mathbf{n}(m)$. Then $g_{p}(\varepsilon)$ is rewritten as

$$
\begin{aligned}
g_{p}(\varepsilon)= & \left\|\mathbf{F}_{p}^{H} \boldsymbol{\Omega}(-\varepsilon) \mathbf{y}(m)-\mathbf{F}_{p}^{H} \boldsymbol{\Omega}(-\varepsilon) \mathbf{y}(m+1) e^{-j 2 \pi \varepsilon K_{s}}\right\|^{2} \\
= & \left(\mathbf{y}^{H}(m)-\mathbf{y}^{H}(m+1) e^{j 2 \pi \varepsilon K_{s}}\right) \boldsymbol{\Omega}(\varepsilon) \mathbf{P}_{p} \boldsymbol{\Omega}(-\varepsilon)(\mathbf{y}(m) \\
& \left.-\mathbf{y}(m+1) e^{-j 2 \pi \varepsilon K_{s}}\right),
\end{aligned}
$$

where $\mathbf{P}_{p}=\mathbf{F}_{p} \mathbf{F}_{p}^{H}$ is the projection matrix onto the subspace spanned by $\mathbf{F}_{p}$. Bearing in mind that $\mathbf{F}_{p}^{H}(\boldsymbol{\eta}(m)-\boldsymbol{\eta}(m+1))=$ 0, $\left.\dot{g}_{p}(\varepsilon)\right|_{\varepsilon=\phi}$ can be obtained as

$$
\begin{aligned}
\dot{g}_{p}(\phi)= & j 2 \pi \Delta \boldsymbol{\eta} \mathbf{D} \mathbf{P}_{p} \Delta \breve{\mathbf{n}}+j 2 \pi \Delta \breve{\mathbf{n}}^{H} \mathbf{D} \mathbf{P}_{p} \Delta \breve{\mathbf{n}} \\
& -j 2 \pi \Delta \breve{\mathbf{n}}^{H} \mathbf{P}_{p} \mathbf{D} \Delta \boldsymbol{\eta}-j 2 \pi \Delta \breve{\mathbf{n}}^{H} P_{p} \mathbf{D} \Delta \breve{\mathbf{n}} \\
& -j 2 \pi K_{s} \boldsymbol{\eta}^{H}(m+1) \mathbf{P}_{p} \Delta \breve{\mathbf{n}} \\
& -j 2 \pi K_{s} \breve{\mathbf{n}}^{H}(m+1) \mathbf{P}_{p} \Delta \breve{\mathbf{n}} \\
& +j 2 \pi K_{s} \Delta \breve{\mathbf{n}}^{H} \mathbf{P}_{p} \boldsymbol{\eta}(m+1) \\
& +j 2 \pi K_{s} \Delta \breve{\mathbf{n}}^{H} \mathbf{P}_{p} \breve{\mathbf{n}}(m+1),
\end{aligned}
$$

where $\mathbf{D} \triangleq \operatorname{diag}\{0,1, \ldots, K-1\}, \Delta \boldsymbol{\eta} \triangleq \boldsymbol{\eta}(m)-\boldsymbol{\eta}(m+1)$ and $\Delta \breve{\mathbf{n}} \triangleq \breve{\mathbf{n}}(m)-\breve{\mathbf{n}}(m+1)$ are used for notation simplicity. The expectation of $\dot{g}_{p}(\phi)$ is

$$
\begin{aligned}
\mathrm{E}\left\{\dot{g}_{p}(\phi)\right\}= & (0)+j 4 \pi \sigma^{2} \operatorname{tr}\left(\mathbf{D} \mathbf{P}_{p}\right)-(0)-j 4 \pi \sigma^{2} \operatorname{tr}\left(\mathbf{P}_{p} \mathbf{D}\right) \\
& +(0)+j 2 \pi K_{s} \sigma^{2} \operatorname{tr}\left(\mathbf{P}_{p}\right)+(0) \\
& -j 2 \pi K_{s} \sigma^{2} \operatorname{tr}\left(\mathbf{P}_{p}\right)=0 .
\end{aligned}
$$

After some manipulations, $\mathrm{E}\left\{\left(\dot{g}_{p}(\phi)\right)^{2}\right\}$ and $\mathrm{E}\left\{\ddot{g}_{p}(\phi)\right\}$ can be obtained as

$$
\begin{aligned}
& \mathrm{E}\left\{\left(\dot{g}_{p}(\phi)\right)^{2}\right\}=16 \pi^{2} \sigma^{2}\left\|\mathbf{P}_{p}\left(\mathbf{D} \Delta \boldsymbol{\eta}-K_{s} \boldsymbol{\eta}(m+1)\right)\right\|^{2}, \\
& \mathrm{E}\left\{\ddot{g}_{p}(\phi)\right\}=8 \pi^{2}\left\|\mathbf{P}_{p}\left(\mathbf{D} \Delta \boldsymbol{\eta}-K_{s} \boldsymbol{\eta}(m+1)\right)\right\|^{2} .
\end{aligned}
$$

On the other hand, $g_{v}(\varepsilon)$ can be rewritten as

$$
\begin{aligned}
g_{v}(\varepsilon) & =\sum_{q=m}^{m+1}\left\|\mathbf{F}_{v}^{H} \boldsymbol{\Omega}(-\varepsilon) \mathbf{y}(q)\right\|^{2} \\
& =\sum_{q=m}^{m+1} \mathbf{y}^{H}(q) \boldsymbol{\Omega}(\varepsilon) \mathbf{P}_{v} \boldsymbol{\Omega}(-\varepsilon) \mathbf{y}(q)
\end{aligned}
$$

where $\mathbf{P}_{v}=\mathbf{F}_{v} \mathbf{F}_{v}^{H}$ is the projection matrix onto the subspace spanned by $\mathbf{F}_{v}$. Bearing in mind that $\mathbf{F}_{v}^{H} \boldsymbol{\eta}(m)=\mathbf{0}$, $\left.\dot{g}_{v}(\varepsilon)\right|_{\varepsilon=\phi}$ can be obtained as

$$
\begin{aligned}
\dot{g}_{v}(\phi)= & j 2 \pi \sum_{q=m}^{m+1}\left(\boldsymbol{\eta}^{H}(q) \mathbf{D} \mathbf{P}_{v} \breve{\mathbf{n}}(q)+\breve{\mathbf{n}}^{H}(q) \mathbf{D} \mathbf{P}_{v} \breve{\mathbf{n}}(q)\right. \\
& \left.-\breve{\mathbf{n}}^{H}(q) \mathbf{P}_{v} \mathbf{D} \boldsymbol{\eta}(q)-\breve{\mathbf{n}}^{H}(q) \mathbf{P}_{v} \mathbf{D} \breve{\mathbf{n}}(q)\right) .
\end{aligned}
$$

It can be calculated that

$$
\begin{aligned}
\mathrm{E}\left\{\dot{g}_{v}(\phi)\right\} & =j 2 \pi \sigma^{2} \sum_{q=m}^{m+1}\left(0+\operatorname{tr}\left(\mathbf{D} \mathbf{P}_{v}\right)+0-\operatorname{tr}\left(\mathbf{P}_{v} \mathbf{D}\right)\right) \\
& =0 .
\end{aligned}
$$

Furthermore, $\mathrm{E}\left\{\left(\dot{g}_{v}(\phi)\right)^{2}\right\}$ and $\mathrm{E}\left\{\ddot{g}_{v}(\phi)\right\}$ can be obtained as

$$
\begin{aligned}
& \mathrm{E}\left\{\left(\dot{g}_{v}(\phi)\right)^{2}\right\}=8 \pi^{2} \sigma^{2} \sum_{q=m}^{m+1} \boldsymbol{\eta}^{H}(q) \mathbf{D P}_{v} \mathbf{D} \boldsymbol{\eta}(q), \\
& \mathrm{E}\left\{\ddot{g}_{v}(\phi)\right\}=8 \pi^{2} \sum_{q=m}^{m+1} \boldsymbol{\eta}^{H}(q) \mathbf{D} \mathbf{P}_{v} \mathbf{D} \boldsymbol{\eta}(q) .
\end{aligned}
$$

Lastly, we derive the expectation of $\dot{g}_{p}(\phi) \dot{g}_{v}(\phi)$ as

$$
E\left\{\dot{g}_{p}(\phi) \dot{g}_{v}(\phi)\right\}=O\left(\mathbf{n}(m)^{4}\right)+O\left(\mathbf{n}(m+1)^{4}\right),
$$

where the property $\mathbf{P}_{p}^{H} \mathbf{P}_{v}=\mathbf{0}$ is used, and $O\left(\mathbf{n}(m)^{4}\right)$ denotes the function at the order of $\mathbf{n}(m)^{4}$. This term can be ignored at higher SNR compared to $E\left\{\dot{g}_{p}(\phi)^{2}\right\}$ and $E\left\{\dot{g}_{v}(\phi)^{2}\right\}$. Therefore, the $p$-algorithm and $v$-algorithm can be considered as uncorrelated to each other.

Finally, substituting (46), (47), (48), (51), (52), (53) into (29), (30) yields (31), (32).

\section{REFERENCES}

[1] J. Bingham, "Multicarrier modulation for data transmission: an idea whose time has come," IEEE Commun. Mag., vol. 28, pp. 5-14, May 1990.

[2] W. Y. Zou and Y. Wu, "COFDM: an overview," IEEE Trans. Broadcasting, vol. 41, pp. 1-8, Mar. 1995.

[3] "Radio broadcasing system, digital audio broadcasting (DAB) to mobile, portable, and fixed receiver," Eur. Telecommun. Stand. Inst., SophiaAntipolis, Valbonne, France, ETS 300 401, 1995-1997.

[4] "Digital broadcasting system television, sound, and data services; framing structure, channel coding, and modulation digital terrestrial television,' Eur. Telecommun. Stand. Inst., Sophia-Antipolis, Valbonne, France, ETS 300 744, 1996.

[5] "Broadband radio access networks (BRAN): high performance radio local area networks (HIPERLAN), type 2; systems overview," Eur. Telecommun. Stand. Inst., Sophia-Antipolis, Valbonne, France, ETR 101 683114,1999 
[6] "Wireless LAN medium access control (MAC) and physical layer (PHY) specifications: high speed physical layer in the 5 GHZ band," IEEE802.11a, 1999.

[7] Y. Li, N. Seshadri, and S. Ariyavisitakul, "Channel estimation for OFDM systems with transmitter diversity in mobile wireless channels," IEEE J. Select. Areas Commun., vol. 17, pp. 461-471, Mar. 1999.

[8] Y. Li, "Simplified channel estimation for OFDM systems with multiple transmit antennas," IEEE Trans. Wireless Commun., vol. 1, pp. 67-75, Jan. 2002.

[9] T. Pollet, M. van Bladel, and M. Moeneclaey, "BER sensitivity of OFDM systems to carrier frequency offset and Wiener phase noise," IEEE Trans. Commun., vol. 43, pp. 191-193, Feb. 1995.

[10] J.-J van de Beek, M. Sandell, and P. O. Borjesson, "ML estimation of time and frequency offset in OFDM systems," IEEE Trans. Signal Processing, vol. 45, pp. 1800-1805, July 1997.

[11] H. Chen and G. J. Pottie, "A comparison of frequency offset tracking algorithms for OFDM," in Proc. GLOBECOM'03, San Francisco, CA, vol. 2, pp. 1069-1073, Dec. 2003.

[12] T. M. Schmidl and D. C. Cox, "Robust frequency and timing synchronization for OFDM," IEEE Trans. Commun., vol. 45, pp. 1613-1621, Dec. 1997.

[13] P. H. Moose, "A technique for orthogonal frequency division multiplexing frequency offset correction," IEEE Trans. Commun., vol. 42, pp. 2908-2914, Oct. 1994.

[14] M. Morelli and U. Mengali, "Carrier-frequency estimation for transmissions over selective channels," IEEE Trans. Commun., vol. 48, pp. 1580-1589, Sept. 2000.

[15] H. Minn, V. K. Bhargava, and K. B. Letaief, "A robut timing and frequency synchronization for OFDM systems," IEEE Trans. Wireless Commun., vol. 2, pp. 822-839, July 2003.

[16] M. Morelli and U. Mengali, "An improved frequency offset estimator for OFDM applications," IEEE Commun. Lett., vol. 3, pp. 75-77, Mar. 1999.

[17] H. Minn and P. Tarasak, "Improved maximum likelihood frequency estimation based on likelihood metric design," in Proc. ICC'05, Seoul, Korea, vol. 4, pp. 2150-2156, May 2005.

[18] H. Minn, P. Tarasak, and V. K. Bhargava, "OFDM frequency offset estimation based on BLUE principle," in Proc. VTC'02, Vancouver, Canada, vol. 2, pp. 1230-1234, Sept. 2002.

[19] H. Minn, X. Fu, and V. K. Bhargava, "Optimal periodic training signal for frequency offset estimation in frequency-selective fading channels," IEEE Trans. Commun., vol. 54, pp. 1081-1096, June 2006.

[20] F. Classen and H. Meyr, "Frequency synchronization algorithms for OFDM systems suitable for communication over frequency selective fading channels," in Proc. VTC'94, Stockholm, Sweden, vol. 3, pp. 1655-1659, June 1994.

[21] X. Fu and H. Minn, "Modified data-pilot-multiplexed scheme for OFDM systems," in Proc. VTC'05-Fall, Dallas, TX, vol. 2, pp. 1011-1015, Sept. 2005.

[22] H. Minn and N. Al-Dhahir, "Estimation of carrier-frequency offset and frequency-selective channels in MIMO OFDM systems using a common training signal," in Proc. WNCG Wireless Networking Symposium, Austin, TX, Oct. 2004.

[23] T. Cui, and C. Tellambura, "Robust joint frequency offset and channel estimation for OFDM systems," in Proc. VTC'04-Fall, Los Angeles, CA, vol. 1, pp. 603-607, Sept. 2004.

[24] G. Santella, "Frequency and symbol synchronization system of OFDM signals: architecture and simulation results," IEEE Trans. Veh. Technol., vol. 49, pp. 254-275, Jan. 2000.

[25] H. Liu, and U. Tureli, "A high-efficiency carrier estimator for OFDM communications," IEEE Commun. Lett., vol. 2, pp. 104-106, Apr. 1998.

[26] B. Chen, "Maximum likelihood estimation of OFDM carrier frequency offset," IEEE Signal Processing Lett., vol. 9, pp. 123-126, Apr. 2002.

[27] F. Gao and A. Nallanathan, "Blind maximum likelihood CFO estimation for OFDM systems via polynomial rooting," IEEE Signal Processing Lett., vol.13, pp. 73-76, Feb. 2006.

[28] H. Minn, Y. Li, N. Al-Dhahir, and R. Calderbank, "Pilot designs for consistent frequency offset estimation in OFDM systems," in Proc. ICC'06, Istanbul, Turkey, vol. 10, pp. 4566-4571, June 2006.

[29] Y. Li, H. Minn, N. Al-Dhahir, and R. Calderbank, "Robust pilot design for consistent carrier frequency offset estimation," in Proc. MILCOM'O6, Washington, DC, pp. 1-7, Oct. 2006.

[30] R. Negi and J. Cioffi, "Pilot tone selection for channel estimation in a mobile OFDM system," IEEE Trans. Consum. Electron., vol. 44, pp. 1122-1128, Aug. 1998.

[31] X. Ma, C. Tepedelenlioglu, G. B. Giannakis, and S. Barbarossa, "Nondata-aided carrier offset estimators for OFDM with null subcarriers: identifiability, algorithms, and performance," IEEE J. Select. Areas Commun., vol. 19, pp. 2504-2515, Dec. 2001.

[32] M. Pesavento, A. B. Gershman, and K. M. Wong, "Direction finding in partly calibrated sensor arrays composed of multiple subarrays," IEEE Trans. Signal Processing, vol. 50, pp. 2103-2115, Sept. 2002.

[33] M. Morelli and U. Mengali, "A comparison of pilot-aided channel estimation methods for OFDM systems," IEEE Trans. Signal Processing, vol. 49, pp. 3065-3073, Dec. 2001.

[34] T. Cui and C. Tellambura, "Low-complexity pilot-aided channel estimation for OFDM systems over doubly-selective channels," in Proc. ICC'05, Seoul, Korea, vol. 3, pp. 1980-1984, May 2005.

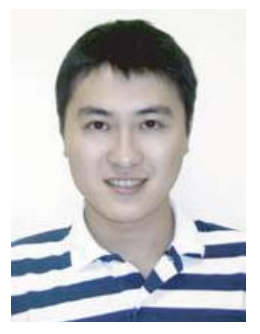

Feifei Gao ( $\left.\mathrm{S}^{\prime} 05\right)$ received the B.Eng. degree in information engineering from Xi'an Jiaotong University, Xi'an, Shaanxi China, in 2002, the M.Sc. degree from the McMaster University, Hamilton, ON, Canada in 2004, and is currently working toward the Ph.D. degree at the Department of Electrical Engineering, National University of Singapore. His research interests are in communication theory, broadband wireless communications, signal processing for communications, MIMO systems, and array signal processing.

Mr. Gao was a recipient of the president scholarship from the National University of Singapore.

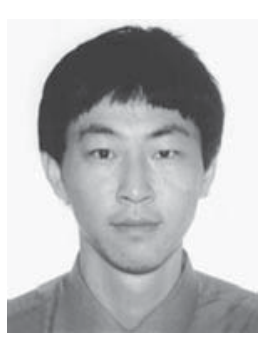

Tao Cui (S'04) received the M.Sc. degree in the Department of Electrical and Computer Engineering, University of Alberta, Edmonton, AB, Canada, in 2005, and the M.S. degree from the Department of Electrical Engineering, California Institute of Technology, Pasadena, USA, in 2006. He is currently working toward the Ph.D. degree at the Department of Electrical Engineering, California Institute of Technology, Pasadena. His research interests are in the interactions between networking theory, communication theory, and information theory.

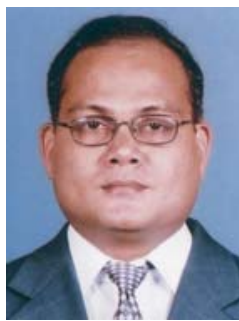

Arumugam Nallanathan (S'97?M'00?SM'05) received the B.Sc. with honors from the University of Peradeniya, Sri-Lanka, in 1991, the CPGS from the Cambridge University, United Kingdom, in 1994 and the Ph.D. from the University of Hong Kong, Hong Kong, in 2000, all in Electrical Engineering. $\mathrm{He}$ was an Assistant Professor in the Department of Electrical and Computer Engineering, National University of Singapore, Singapore from August 2000 to December 2007. Currently, he is a senior lecturer at King's College London. His research interests include OFDM systems, ultra-wide bandwidth (UWB) communication and localization, MIMO systems, and cooperative diversity techniques. In these areas, he has published over 100 journal and conference papers. He is a co-recipient of the Best Paper Award presented at 2007 IEEE International Conference on Ultra-Wideband.

He currently serves on the Editorial Board of IEEE Transactions on Wireless Communications, IEEE Transactions on Vehicular Technology, JohnWiley's Wireless Communications and Mobile computing and EURASIP Journal of Wireless Communications and Networking as an Associate Editor. He served as a Guest Editor for EURASIP Journal of Wireless Communications and Networking: Special issue on UWB Communication Systems- Technology and Applications. He also served as a technical program committee member for more than 25 IEEE international conferences. He currently serves as the General Track Chair for IEEE VTC'2008-Spring, Co-Chair for the IEEE GLOBECOM'2008 Signal Processing for Communications Symposium, and IEEE ICC'2009 Wireless Communications Symposium. 